\title{
Time-Resolved X-ray Photoabsorption and Diffraction on Timescales from ns to fs
}

\author{
P.A. Heimann ${ }^{1}$, T. Missalla ${ }^{2}$, A. Lindenberg ${ }^{3}$, I. Kang ${ }^{3}$, S. Johnson ${ }^{3}$, \\ Z. Chang ${ }^{4}$, H.C. Kapteyn ${ }^{4}$, R.W. Lee ${ }^{2}$, R.W. Falcone ${ }^{3}$, R.W. Schoenlein ${ }^{5}$, \\ T.E. Glover ${ }^{1}$, A.A. Zholents ${ }^{6}$, M.S.Zolotorev ${ }^{6}$ and H.A. Padmore ${ }^{1}$ \\ ${ }^{1}$ Advanced Light Source, Lawrence Berkeley National Laboratory, Berkeley, CA 94720 \\ ${ }^{2}$ Lawrence Livermore National Laboratory, Livermore, CA 94551 \\ ${ }^{3}$ Physics Department, University of California at Berkeley, Berkeley, CA 94720 \\ ${ }^{4}$ Center for Ultrafast Optical Science, University of Michigan, Ann Arbor, MI 48109 \\ ${ }^{5}$ Materials Sciences Division, Lawrence Berkeley National Laboratory, Berkeley, CA 94720 \\ ${ }^{6}$ Center for Beam Physics, Accelerator and Fusion Research Division, Lawrence Berkeley National \\ Laboratory, Berkeley, CA 94720
}

\begin{abstract}
Beamline 7.3.3 at the Advanced Light Source combines a femtosecond laser system with an $\mathrm{x}$ ray streak camera. Time-resolved $x$-ray diffraction with picosecond time resolution is used to observe scattering from coherent acoustic phonons in laser-excited InSb crystals. Synchrotron radiation pulses of $\sim 300 \mathrm{fs}$ in duration have been generated by femtosecond laser pulses modulating the electron beam in the Advanced Light Source.
\end{abstract}

\section{INTRODUCTION}

Time-resolved $x$-ray photoabsorption and diffraction enable one to probe the electronic and structural changes associated with phase transitions, solid state dynamics and chemical reactions. Important structural dynamics occur on the timescales from nanoseconds to femtoseconds with one fundamental limit set by vibrational periods, $\sim 100 \mathrm{fs}$. Recent experiments, using both synchrotron and laserplasma based sources, have observed phase transitions and chemical reactions on picosecond time-scales. In biology, real-time studies of photo-initiated reactions in complex molecules such as photoactive yellow protein (PYP) have been performed (1). Diffraction experiments utilizing laser-plasma $X$-ray sources have observed laserinduced disorder in Langmuir-Blodgett films (2) and a coherent acoustic pulse in GaAs (3).

Two approaches are being pursued at the Advanced Light Source (ALS): the first utilizing ultrafast detectors and the second developing a femtosecond $\mathrm{x}$-ray source. At 
ALS beamline 7.3.3 $\mathrm{x}$-rays are detected by a streak camera with picosecond time resolution. The long range order of laser-irradiated InSb has been probed by the $\mathrm{x}$-ray diffraction. Oscillations in the InSb crystal reflectivity are observed because of scattering from coherent acoustic phonons. A femtosecond $\mathrm{x}$-ray source has been produced by co-propagating a femtosecond laser pulse with an electron bunch in a wiggler. In recent proof-of-principle experiments, we have successfully generated 300 fs synchrotron pulses for the first time.

\section{ALS BEAMLINE 7.3.3, LASER, STREAK CAMERA}

The ALS storage ring is operated in several timing modes, two of which are suitable for time-resolved experiments. In double-bunch mode, only two buckets are filled with a separation of $328 \mathrm{~ns}$. During 'camshaft' operation a series of buckets are filled 2 ns apart, and one additional electron bunch is placed with a $32 \mathrm{~ns}$ gap before and afterwards. An individual $\mathrm{x}$-ray pulse is $\sim 50 \mathrm{ps}$ long.

ALS beamline 7.3.3 has a toroidal mirror, which provides a 1:1 double focus of the bend magnet source in the $\mathrm{x}$-ray hutch. The focus was measured to be $200 \mu \mathrm{m}$ horizontally x $70 \mu \mathrm{m}$ vertically (fwhm), corresponding to a tangential slope error of 1 $\mu \mathrm{rad}$ rms. The photon energy range extends from $1.8 \mathrm{keV}$ (determined by a $50 \mu \mathrm{m}$ thick Be window) to about $12 \mathrm{keV}$. A differential pumping section has been fabricated, which will bring the lower photon energy limit to $\sim 500 \mathrm{eV}$. Inside the hutch a chopper with $1 \mathrm{kHz}$ frequency reduces the average power by a factor of 20 . The final beamline component is a two crystal monochromator, which uses alternatively $\mathrm{Si}(111), \mathrm{Ge}(111)$ or InSb (111) crystals. The second crystal translates to maintain a constant exit beam height.

The laser system consists of a $\mathrm{Ti}_{2} \mathrm{Al}_{2} \mathrm{O}_{3}$ laser oscillator pumped by a frequencydoubled $\mathrm{Nd}$-laser, and a $\mathrm{Ti}_{\mathrm{A}} \mathrm{Al}_{2} \mathrm{O}_{3}$ regenerative amplifier and multipass amplifier operating at a repetition rate of $1 \mathrm{kHz}(4)$. The pulse duration is $150 \mathrm{fs}$, and the pulse energy is $2 \mathrm{~mJ}$. The laser pulses are synchronized with the synchrotron radiation pulses by locking the laser-oscillator repetition frequency to the master RF clock of the ALS. The jitter between the laser and the synchrotron radiation pulses is estimated to be about 5 ps.

The sample is mounted in vacuum on a one-circle goniometer with $\mathrm{XYZ}$ translation. It is possible to cool the sample with a liquid helium cryostat.

The $\mathrm{x}$-rays are detected by a streak camera (5). The streak camera consists of a CsI photocathode, an anode slit, deflection plates, a magnetic lens, a microchannel plate intensifier, a phosphor screen and a fiber-coupled CCD. A high voltage pulse to the microchannel plate intensifier provides a gate for individual $\mathrm{x}$-ray pulses. A singleshot time resolution of $540 \mathrm{fs}$ has been demonstrated. In averaging mode a GaAs photoconductive switch drives the high-voltage pulse to the deflection plates. Using UV pulses from the laser, an effective time resolution of 2 ps was observed. The streak camera has a quantum efficiency of about $1 \%$ limited by the difference between the $\mathrm{x}$-ray absorption and electron escape depths of photocathode materials. 


\section{TIME-RESOLVED X-RAY DIFFRACTION OF INSB}

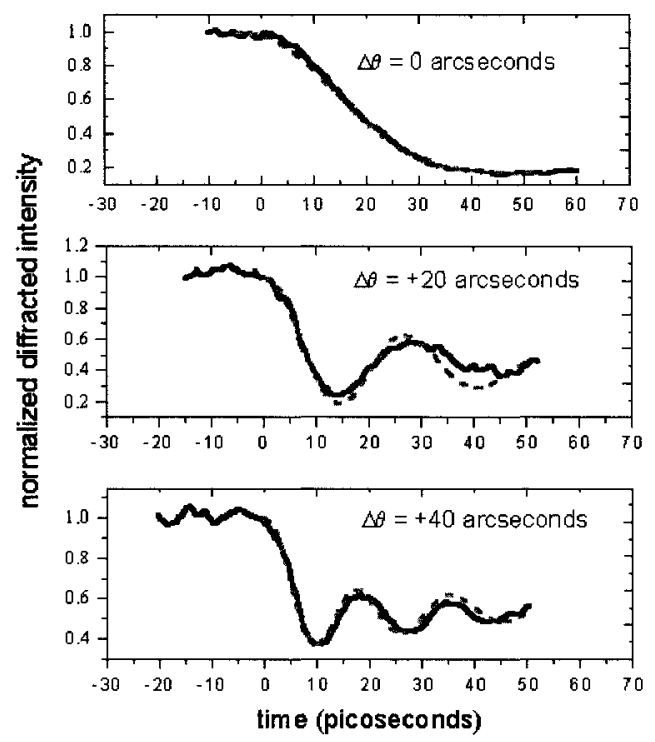

FIGURE 1. Experimentally measured (solid line) and simulated (dashed line) time-resolved diffracted intensity at crystal angles of $0,+20$, and +40 areseconds from the Bragg peak.

The (111) reflection from laser-irradiated InSb was studied at an $\mathrm{x}$-ray wavelength of $2.4 \AA$. To better match the penetration depths of the laser and $x$-rays, the crystal is asymmetrically-cut so that the diffracted beam leaves the crystal at a grazing angle of about 3 degrees. Figure 1 shows the time-dependent diffracted intensity measured at 0 , +20 , and +40 arcseconds from the Bragg peak. The laser fluence was $20 \%$ below the damage threshold of $15 \mathrm{~mJ} / \mathrm{cm}^{2}$. Impulsive excitation of a solid on a timescale shorter than the material's acoustoelastic response time generates coherent acoustic phonons across a range of wavevectors peaked at a wave-number of order one inverse laser penetration depth $(100 \mathrm{~nm})$. As the crystal angle is varied, different phonon modes are probed out of the spectrum of excited modes. By wavevector matching considerations, the phonon frequency $\omega$ observed at a deviation $\Delta \theta$ from the Bragg angle $\theta$ of a symmetric crystal reflection is given by

$$
\omega=\mathrm{v}|\mathbf{G}| \Delta \theta \cot \theta \text {, }
$$

where $v$ is the speed of sound within the crystal and $\mathbf{G}$ the reciprocal lattice vector.

Figure 1 includes calculated (normalized) diffracted intensities based on dynamical diffraction theory coupled to analytic solutions for the laser-induced strain profile. For an instantaneously heated crystal with assumed exponential temperature profile near 
the surface, the time-dependent strain profiles have been derived by Thomsen et al (6). There are three adjustable parameters in our model: the time for thermal transfer of energy to the lattice and the amplitudes of the thermal and deformation potential generated strain. The best fits correspond to a thermal energy transfer time of $12 \mathrm{ps,a}$ thermal strain of $0.17 \%$ (just below that of InSb at its melting temperature) and a nonthermal contribution a factor of two smaller. At a slightly higher laser fluence, $10 \%$ below the damage threshold, no temporal oscillations occur.

\section{A FEMTOSECOND X-RAY SOURCE}

The technique for generating femtosecond $\mathrm{x}$-rays from the ALS is based on extracting a $\sim 100$ fs slice of the long (30 ps) electron bunch using a femtosecond laser pulse. (7) Co-propagation of a femtosecond laser pulse with the electron bunch through an appropriately tuned wiggler results in acceleration (and deceleration) of an ultrashort slice of electrons. These electrons will separate spatially from the main electron bunch in a dispersive section of the storage ring. Radiation from the modulated electrons at a bend magnet source can be imaged onto a slit isolating the ultrafast $\mathrm{x}$-ray pulse.

The laser and wiggler emission in the near and far fields are overlapped using diagnostics at the wiggler front end. The spectrum of the laser is also matched to the fundamental of the wiggler. The efficiency of the interaction between the laser and electron beam is tested by measuring the gain in the laser pulse energy.

Femtosecond duration synchrotron pulses are directly measured by cross-correlating the visible light from bend-magnet beamline (6.3.2) at the ALS with the synchronized laser pulses in a non-linear crystal. Figure 2(a) shows a laser synchrotron crosscorrelation measurement on a long time scale. The measured pulse duration, $39 \mathrm{ps}$ FWHM, corresponds to the overall electron bunch duration. Measurement with higher
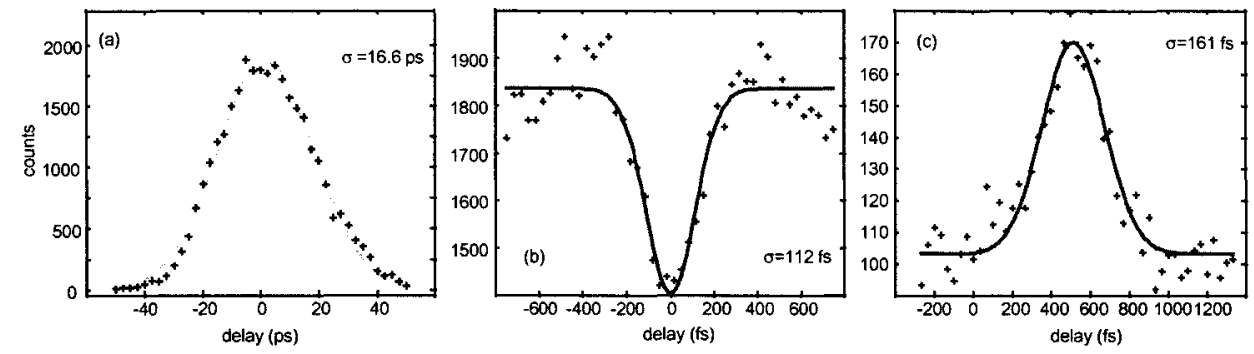

FIGURE 2. Cross correlation of the visible synchrotron pulse with a femtosecond laser pulse: (a) overall synchrotron pulse, (b) femtosecond dark pulse from on-axis radiation, (c) femtosecond pulse from off-axis radiation.

time resolution (Fig. 2b) shows the femtosecond "dark" pulse ( $260 \mathrm{fs}$ FWHM) originating from the central core of the electron bunch. Figure 2(c) shows a 
measurement with a knife edge at the $3 \sigma_{\mathrm{x}}$ position, where the femtosecond "bright" pulse is observed ( $380 \mathrm{fs}$ FWHM). The femtosecond time structure will be invariant over the entire spectral range of bend-magnet emission from the near infrared to $\mathrm{x}$-ray wavelengths. The observed pulse duration is limited by the position of the bend magnet beamline used in these characterization measurements. For a bend magnet immediately following the wiggler, an $\mathrm{x}$-ray pulse duration of $\sim 100 \mathrm{fs}$ is calculated.

\section{ACKNOWLEDGMENTS}

This work was supported by the U.S. Department of Energy through Lawrence Berkeley National Laboratory and the Institute for Laser Science and Applications at Lawrence Livermore National Laboratory. The ALS is supported by the Director, Office of Science, Office of Basic Energy Sciences, Materials Sciences Division of the U.S. Department of Energy under Contract No. DE-AC03-76SF00098.

\section{REFERENCES}

1. Perman, B., Srajer, V., Ren, Z., Teng, T., Pradervand, C., Ursby, T., Bourgeois, D., Schotte, F., Wulff, M., Kort, R., Hellingwerf, K., Moffat, K., Science 279, 1946-50 (1998).

2. Rischel, C., Rousse, A., Uschmann, I., Albouy, P.-A., Geindre, J.-P., Audebert, P, Gauthier, J.C., Forster, E., Martin, J.L.,Antonetti, A., Nature 390, 490-492 (1997).

3. Rose-Petruck, C., Jimenez, R., Guo, T., Cavalleri, A., Siders, C.W., Raksi, F., Squiers, J.A., Walker, B.C., Wilson, K.R., Barty, C.P.J., Nature 398, 310-312 (1999).

4. Larsson, J., Chang, Z., Judd, E., Schuck, P. J., Falcone, R. W., Heimann, P. A., Padmore, H. A., Kapteyn, H. C., Bucksbaum, P.H., Murnane, M. M., Lee, R. W., Machacek, A., Wark, J. S., Liu, X., and Shan, B., Optics Lett. 22, 1012-1014 (1997).

5. Chang, Z., Rundquist, A., Zhou, J., Murnane, M.M., Kapteyn, H.C., Liu, X., Shan, B., Liu, J., Niu, L., Gong, M., Zhang, X., Appl. Phys. Lett. 69, 133-135 (1996).

6. Thomsen, C., Grahn, H.T., Maris, H.J., and Tauc, J., Phys. Rev. B 34, 4129-4138 (1986).

7. Zholents, A.A., Zolotorev, M.S., Phys. Rev. Lett. 76, 912-915 (1996). 